066.

\section{Automated Defibrillation by Police First-Responders: Device and Operator Performance}

Vincent N. Mosesso, Jr., MD, FACEP, Michael Baumann, MD, * Eric Davis, MD, FACEP, Mary Ann Scott, RN, BSN, EMT-P, Raymond E. Carlin, BS, EMT-P, William Plunkett, Med, EMT-P University of Pittsburgh, Division of Emergency Medicine, Pittsburgh, Pennsylvania USA

Purpose: We sought to describe device and operator performance during a three-year study of automatic external defibrillator (AED) use by police first-responders.

Methods: This was a prospective, consecutive case series of AED applications in seven suburban communities (population $=142,000$ ). Two hundred police first-responders were trained to use the LifePak $300^{\circledR}$ semi-automatic defibrillator. We included AED uses by police from February 1992 through January 1995 . We collected data from dispatch logs, EMS patient records, AED voice and EKG recordings, and police and EMS study data sheets.

Results: Police applied the AED to 128 patients. Four uses were excluded due to insufficient data. Three uses were inappropriate (two patients with pulses; one with major trauma). The AED failed to advise shock of VF in four cases

\section{Operator Performance}

Critical Action

Correct/Total

Airway management

$106 / 124 \quad(85 \%)$

Pad placement

Analysis within $2 \mathrm{~min}$. of arrival

Shock within 30 secs. of prompt

"Clear" patient before shcok

$120 / 124 \quad(97 \%)$

$102 / 124 \quad(82 \%)$

$81 / 81 \quad(100 \%)$

$81 / 81 \quad(100 \%)$

Re-analysis within 15 seconds of shock

$81 / 81 \quad(100 \%)$

CPR if "no shock" advised

$83 / 124 \quad(67 \%)$

\section{Device Performance}

Critical Action

Correct/Total

Mechanical function

Properly advise shock

(96\%)

Properly advise "no shock"

$81 / 85 \quad(95 \%)$

256/256 (100\%)

$338 / 339 \quad(99 \%)$

Conclusion: We found a low but significant number of AED device errors. Police performed six of seven critical actions properly $>80 \%$ of attempts. Training should emphasize doing CPR after "no shock" advisory and prompt initial analysis. Defibrillation by police is feasible in selected communities.

065.

\section{Law Enforcement Agencies and Out-of- Hospital Emergency Medical Care: A National Survey}

Hector M. Alonso-Serra, MD, * Theodore Delbridge, $M D, M P H$, Thomas Auble, PhD, Vincent Mosesso, MD, Eric Davis, $M D$

Division of Emergency Medicine, University of Pittsburgh, Pittsburgh, Pennsylvania USA

Purpose: Previous studies have demonstrated that law enforcement officers can be trained successfully to use automatic external defibrillators (AED) and reduce response time to cardiac arrests. We sought to determine the current level of interest and involvement by law enforcement agencies in working together with emergency medical services (EMS) to enhance out-of-hospital resuscitation capabilities.

Methods: A 20-question survey was mailed nationwide to 800 randomly selected police chiefs and sheriffs from an American Police Hall of Fame and Museum mailing list. Survey questions requested community and EMS system descriptions, indications of current agency EMS involvement, and attitudes regarding increased involvement as EMS first-responders.

Results: Of the 800 surveys mailed, 17 were returned by the post office, resulting in $783(\mathrm{~N})$ received by the addressee. We received 414 completed surveys $(53.9 \%)$. The respondents served a total population of $12,612,420$. The median population served was $6,000(\min =260, \max =1.5$ million $)$. Responses indicated that $241(58.2 \%)$ agencies routinely respond to some emergency medical situations and that $161(38.9 \%)$ provide some level of care. Only eight $(1.9 \%)$ of the respondents currently employ AEDs, yet $56(13.5 \%)$ indicated that in the majority of cases, they have a shorter response time than EMS. Each of these 56 respondents also expressed willingness to receive more training and expand their current role in EMS. Enhanced public image was thought to be a benefit of EMS-related activities by $253(61.1 \%)$ of respondents.

Conclusion: The survey results indicate that there is substantial involvement of law enforcement agencies in out-of-hospital EMS and that most agencies would support additional training and an increased role in EMS. 